\title{
Cognitive Radio With Software Defined Radio and Mimo for Future Generation Wireless Communication
}

\author{
Shashank Singh ${ }^{1 *}$, Gowher Mushtaq ${ }^{1}$, Neeraj Kumar Tiwari ${ }^{1}$ and Akhand Pratap Singh ${ }^{2}$ \\ ${ }^{1}$ Computer Science and Engineering, Shri Ramswaroop Memorial University, Lucknow, India \\ ${ }^{2}$ Graphic Era University, Dehradun, Uttarakhand, India
}

\begin{abstract}
Spectrum is a natural resource of communication path and it is main entity of wireless communication. At present maintain the spectrum secrecy is a big problem because of exponential growth of users and devices of wireless communication. Spectrum have two types of user first one is primary i.e. licenced and other one is secondary i.e. unlicensed user. Secondary user doesn't need any license to operate but primary user needs to license to operate in a fix geographical area with a fixes time duration. The entire time spectrum is underutilized. Spectrum has limited frequencies so we can't increase spectrum frequencies but we try to improve spectrum efficiency by the help of different technologies and methodologies. Cognitive radio, Software defined Radio and Spectrum sharing technique play an important role to improve spectrum efficiency but interference, false alarm and low detection is a problem that can reduce by MIMO technology. In this paper we discuss about cognitive radio network and Software Defined Radio technology to improve the performance of wireless communication and MIMO technology to reduce interference and false alarm and improve probability of detection to build future generation of wireless communication.
\end{abstract}

Keywords: Cognitive radio; SDR; Spectrum secrecy; Spectrum efficiency; MIMO; Spectrum sharing

\section{Introduction}

Wireless communication system works on frequencies provided by spectrum. Spectrum has limited frequencies because it is a natural resource. Wireless devices communicate on different frequencies ranges. Frequency band is categories on the basis of ranges of frequency. Every band has different application areas. According to Institute of Electrical and Electronics Engineers (IEEE) standard frequencies divided in many bands i.e. HF, VHF, UHF, L, S, C, X, Ku, K, Ka, V, $\mathrm{W}, \mathrm{mm}$ shown in Table 1 [1]. As we see in current scenario number of user of wireless communication system increases dramatically because it is easy to access any time anywhere and also beneficial in many aspects than wired communication. According to Wireless World Research Forum by year 2017 approximate Seven trillion wireless devices will serve seven billion people [2]. User of spectrum of two types' i.e. licensed and unlicensed and it is not necessary that licensed user use spectrum every time [3]. Cognitive radio network is a technology that sense unused frequencies by licensed user and provide unused frequency to unlicensed user. Concept of cognitive radio is first introduced by Joe Mitola in 1999-2000 so sometimes it also called Mitola radio and defined by Haykins, main objective of cognitive radio is to provide reliability by help of capability of awareness of surrounding, learning and adaptability and change parameter in real time. Cognitive radio divided into two type's first one is full cognitive radio and other one is spectrum sensing cognitive radio [1,3-5]. One more reason to think about efficient utilization of spectrum is next generation wireless application need to high transmission capacity and speed. But in previous licensing scheme of spectrum does not allow to one licensed can't change the type of use or on licensed user can't transfer the right to other licensed user. Basically two types of spectrum sharing tech are present i.e. static spectrum allocation and dynamic spectrum access. Lack of interoperability, faces difficulty by advancement of old technology, normally spectrum is underutilized is main drawbacks of static spectrum allocation technique so to overcome these drawbacks dynamic spectrum access tech is introduced by radio engineers dynamic spectrum access is most efficient way of spectrum sharing [3]. In dynamic spectrum access spectrum is dynamically accessed on the basis of users need. We can say that dynamic spectrum access is a synonym for cognitive radio because dynamic spectrum access is an important task of cognitive radio. Cognitive radio uses dynamic spectrum access technique to find white space [5-7] i.e. unused frequencies (Table 1).

\section{Cognitive radio}

Cognitive radio is a powerful concept. However under some barriers it is possible to build a network of radios that means nodes by linking various nodes of cognitive radio. In this way several elements of the performance can be considerably enhanced. A single cognitive radio will communicate with several non-cognitive radio stations like femto-cell requires cognitive functionality to communicate with non-cognitive cell-phones at many instances. Cognitive radios will be grouped and able to form a network and act as an overall cognitive radio network and by this network it is possible to obtain the outstanding set of advantages in terms of spectrum sensing and it is possible to retransmit data from one channel to the next so by retransmitting data from one channel to next improve coverage and make energy efficient $[4,6,8]$. As we see in our day to life lot of cellular companies are using cognitive radio for the proper development of the number of applications, the area of spectrum sensing has become increasingly more important. As cognitive radio technology is being used to provide a basic and most suitable method of using the spectrum more intelligently, spectrum sensing is a clue to this application.

Cognitive radio spectrum sensing system must be able to sense,

${ }^{*}$ Corresponding author: Shashank Singh, Faculty of Computer Science and Engineering, Shri Ramswaroop Memorial University, Lucknow, India, Tel: 9005670664; E-mail: shanky197@gmail.com

Received March 17, 2015; Accepted April 07, 2015; Published April 09, 2015

Citation: Singh S, Mushtaq G, Tiwari NK, Singh AP (2015) Cognitive Radio With Software Defined Radio and Mimo for Future Generation Wireless Communication. J Comput Sci Syst Biol 8: 166-169. doi:10.4172/jcsb.1000184

Copyright: ( 2015 Singh S, et al. This is an open-access article distributed under the terms of the Creative Commons Attribution License, which permits unrestricted use, distribution, and reproduction in any medium, provided the original author and source are credited. 


\begin{tabular}{|c|c|c|c|}
\hline Frequency Band & Frequency Range & Wavelength Range & Application \\
\hline $\mathrm{HF}$ & 3-30 MHz & $10-100 \mathrm{~km}$ & Aviation communication, government time stations, weather stations \\
\hline VHF & $30-300 \mathrm{MHz}$ & $1-10 \mathrm{~km}$ & Radio modems, amateur radio, and marine communications \\
\hline UHF & $300-1000 \mathrm{MHz}$ & $100 \mathrm{~m} / \mathrm{km}$ & $\begin{array}{c}\text { Television broadcasting, cordless phones, walkie talkies, personal radio } \\
\text { services, satellite communication }\end{array}$ \\
\hline L & $1-2 \mathrm{GHz}$ & $15-30 \mathrm{~cm}$ & Cellphone, Navigation, Satellite \\
\hline $\mathrm{S}$ & $2-4 \mathrm{GHz}$ & $7.5-15 \mathrm{~cm}$ & Unlicensed (Bluetooth, Wi-Fi etc.), Satellite, Cellphones \\
\hline $\mathrm{C}$ & 4-8 GHz & $3.75-7.5 \mathrm{~cm}$ & Microwave relay, Satellite \\
\hline $\mathrm{X}$ & 8-12 GHz & $2.5-3.75 \mathrm{~cm}$ & Radar \\
\hline $\mathrm{KU}$ & $12-18 \mathrm{GHz}$ & $1.67-2.5 \mathrm{~cm}$ & Satellite TV, Police radar \\
\hline $\mathrm{K}$ & $18-27 \mathrm{GHz}$ & $1.11-1.67 \mathrm{~cm}$ & Microwave backhaul \\
\hline KA & $27-40 \mathrm{GHz}$ & $0.75-1.11 \mathrm{~cm}$ & Microwave backhaul \\
\hline V & $40-75 \mathrm{GHz}$ & $0.4-0.75 \mathrm{~cm}$ & Experimental, radar, New WLAN \\
\hline W & $75-110 \mathrm{GHz}$ & $0.27-0.4 \mathrm{~cm}$ & Automotive radar \\
\hline M & $30-300 \mathrm{GHz}$ & $1 \mathrm{~cm}-1 \mathrm{~mm}$ & Personal area networks \\
\hline
\end{tabular}

Table 1: Frequencies their ranges and applications.

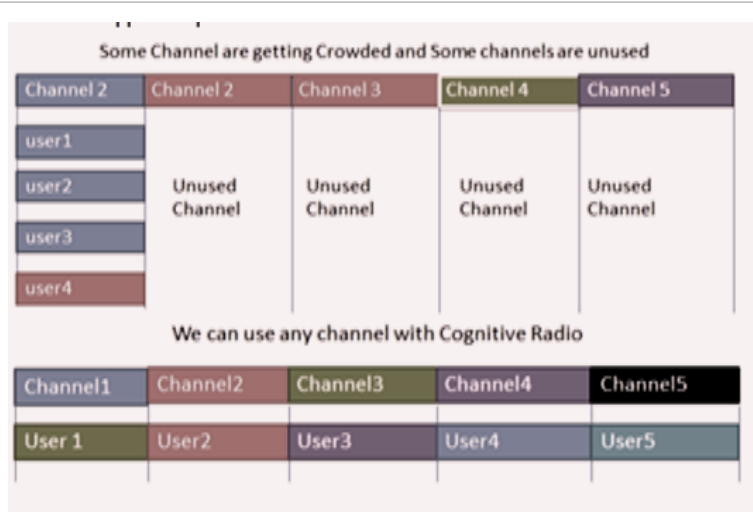

Figure 1: Cooperative spectrum sensing.

monitor and identify any other transmissions and inform to CPU to take suitable action. There are some methods for cognitive radio spectrum sensing like waveform-based sensing, energy detector based sensing, cyclostationarity-based sensing, matched-filtering technique etc $[6,9]$. As per literature review we can say that waveform based sensing is best among these sensing methods. There are two types of spectrum sensing of cognitive radio first one is non-cooperative spectrum sensing and second one is cooperative spectrum sensing. In non-cooperative spectrum sensing it configure itself according to preloaded information and signals $[8,10]$.

Cooperative spectrum sensing has two approaches for sensing first one is centralized and other one is distributed. In first approach a master node concept that stores information from all nodes in network master node have all the control but in second approach no master node concept and no one node take control all nodes share their sense information. Cooperative spectrum sensing is beneficial over non-cooperative spectrum sensing. It removes hidden node problem, increase agility, reduce false information, more accurate signal detection etc $[11,12]$ (Figure 1).

\section{Software defined radio}

SDR stands for software defined radios; concept behind SDR is that the radio can be fully configurable by software. A software use to modify or change the configuration of radios for the function required at given time. On cellular base station a frequent standards upgrades occur so it is an application area for SDR and it is also applicable in commercial world. Main advantage of SDR is to provide reconfigurable it and another is wave form portability and benefits of wave form portability are cost saving, interoperability, and obsolescence mitigation. It contains a number of functional blocks like radio frequency amplification block, Frequency conversion block, and digital conversion base band processor. Basically two types of radio containing software in fashion first one is software controlled radio and second one is software defined radio. Use of software processing within the radio system in software controlled and in software defined radio all the function of its lower level or physical layer is defined by software if any changes occur in software then function of lower layer will also change and in software controlled radio. The most powerful property of SDR is versatility. Main goals of SDR engineers is to facilitates a single radio transceiver is able to playing the roles of telephone, mobile phone, wireless fax, pager, wireless web browser, global positioning system unit, and many other functions operable from any corner of earth surface. SDR also provides many facilities like limitation of hardware, provides radio functionality as software module, flexibility, degree of freedom in radio domain $[6,8,9]$.

\section{Cognitive radio with software defined radio}

One of the most common topics of cognitive radio is spectrum management. This topic centralized on how regulatory database perform externally dynamic intelligence, within receivers, can use spectrum more efficiently than under conventional spectrum management systems. It is attached with software-defined radio (SDR), where the radio's key operating are defined largely in software. SDR along with software defined antennas are the enablers of the cognitive radio. Cognitive radio requires an expandable radio device and SDR provides platform for cognitive radio. Cognitive radio is comprised of a control layer on "top" of an agile software defined radio. Combination of a cognitive "engine" and the SDR together comprise a "cognitive radio". Cognitive cycle contains five phase's i.e. sensing, awareness, learning, adaption, and response that are shown in Figure $2[8,10,11]$.

\section{MIMO in Cooperative Cognitive radio}

In cognitive radio network primary user enrol the secondary user to adopt primary traffic primary user share some part of channel access time to secondary user for cooperation in data exchange. It minimizes the performance of both. Common problem is interference, false alarm, low probability of detection in the cognitive radio network. MIMO that suppresses problems via pre-coding and improves performance of network $[7,12]$. MIMO is an antenna technology that provides multipath propagation i.e. transmitted signal reaches 
Citation: Singh S, Mushtaq G, Tiwari NK, Singh AP (2015) Cognitive Radio With Software Defined Radio and Mimo for Future Generation Wireless Communication. J Comput Sci Syst Biol 8: 166-169. doi:10.4172/jcsb.1000184

\begin{tabular}{|c|c|c|c|c|}
\hline & & \multicolumn{3}{c}{ CR interference-to-noise ratio (dB) } \\
\hline & Average & -40 & -20 & $\mathbf{0}$ \\
\hline \multirow{2}{*}{$\begin{array}{c}\text { Interference at primary } \\
\text { user (dBm) }\end{array}$} & Full Projection Pre-coding & -47.5 & -27.5 & -23 \\
\cline { 2 - 5 } & Sensing Projection Pre-coding & -47.5 & -27.5 & -25 \\
\cline { 2 - 5 } & Partial Projection Pre-coding & -47.5 & -27.5 & -23 \\
\hline
\end{tabular}

Table 2: Average interference in different pre-coding scheme with respect to CR interference to noise ratio.

\begin{tabular}{|c|c|c|c|c|c|}
\hline & . & \multicolumn{4}{|c|}{ CR interference-to-noise ratio (dB) } \\
\hline \multicolumn{2}{|c|}{ Average } & -40 & -20 & 0 & 20 \\
\hline \multirow{3}{*}{$\begin{array}{l}\text { Interference at primary } \\
\text { user }(\mathrm{dBm})\end{array}$} & Full Projection Pre-coding & 21.5 & 21.45 & 20.5 & 19.2 \\
\hline & Sensing Projection Pre-coding & 20 & 19.9 & 19 & 17.8 \\
\hline & Partial Projection Pre-coding & 21.5 & 21.5 & 20.55 & 19. 4 \\
\hline
\end{tabular}

Table 3: Average throughput in different pre-coding scheme with respect to CR interference to noise ratio.

\begin{tabular}{|l|c|c|c|c|c|c|c|c|c|c|}
\hline \multicolumn{10}{|c|}{ Number of Samples } \\
\hline Probability of detection & $\mathbf{0}$ & $\mathbf{1}$ & $\mathbf{2}$ & $\mathbf{3}$ & $\mathbf{4}$ & $\mathbf{5}$ & $\mathbf{1 0}$ & $\mathbf{1 5}$ & $\mathbf{2 0}$ & 1 \\
\hline Probability of false alarm & 0 & 0 & 0 & 0.5 & 0.84 & 0.96 & 1 \\
\hline
\end{tabular}

Table 4: Probability of detection and false alarm with respect to number of samples, where number of antenna is 6 .

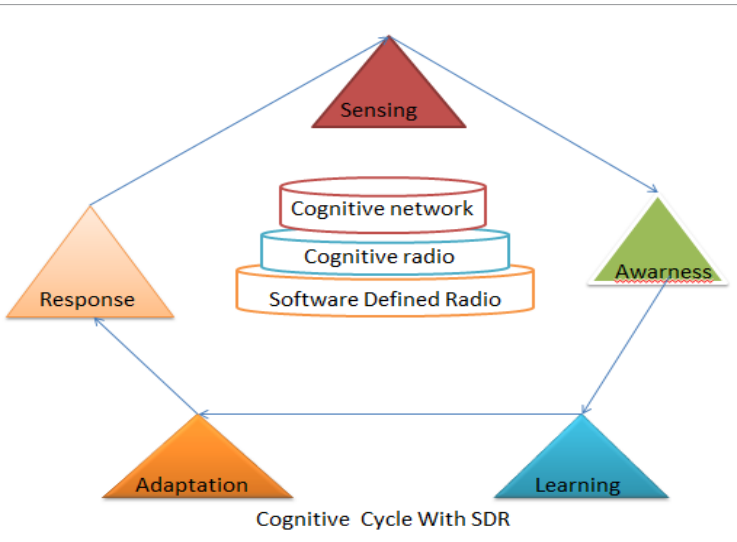

Figure 2: Cognitive Cycle.

at receiver end after travelling many paths. MIMO have three stages pre-coding, spatial multiplexing, diversity coding, pre-coding is a multistream beam forming. Cognitive system generally used for feat unused frequencies of primary spectrum. Transmit beam forming and pre-coding technique is effective for reduce interference of Primary network. Sensing projection based pre-coding is most practical precoding scheme $[13,14]$. It is not useful for reduce interference between primary transmitters to cognitive receiver. So two more improved and effective sensing projection based pre-coding scheme first one is full projection and second is partial projection based pre-coding via sensing these scheme reduce interference of primary cognitive radio. Removal of interference in full projection via sensing and this technique improve flexibility and throughput. On the basis of literature Tables 2 and 3 shows that partial projection improve throughput of cognitive radio transmission a subspace that partially span the estimated null space of the cognitive radio primary interference channel so cognitive radio throughput further increases [15-17].

Increase in probability of detection of signals and degradation in probability of false alarm (Table 4 Where number of antenna=6) in spectrum sensing is achieved by integrating MIMO technology with cognitive radio. The Capacity will also increase with the number of antennas increase [5].

\section{Conclusion}

On the basis of litrature study and analysis we conclude that cognitive radio network provides free channel by the primary user to channel access for secondary user and software defined radio provides platform for cognitive radio networks MIMO technology reduce interference and probability of false alarm in network, improve throughput and signal's probability of detection by the help of sensing based pre-coding scheme i.e full projection and partial projection so we can propose these technologies or methodologies in respect to future generation.

\section{Acknowledgement}

We are thankful to the Faculty members of Computer Science and Engineering, Shri Ramswaroop University for their motivation and continuous support. Our special vote of thanks to Dr. Bineet Gupta for his valuable suggestions and contributions.

\section{References}

1. Radio spectrum, Available from: http://en.wikipedia.org/wiki/Radio_spectrum.

2. Lene S, Knud ES (2009) User scenarios 2020 - a worldwide wireless future WWRF Outlook, Outlook Visions and research directions for the Wireless World 4:1-38.

3. Partha PB, Ronak K, Rishita G, Anjali A (2011) Smart Radio Spectrum Management for Cognitive Radio. International Journal of Distributed and Parallel Systems (IJDPS) 2: 12-24.

4. Ian FA, Lee WY, Mehmet CV, Shantidev M (2006) NeXt generation/dynamic spectrum access/cognitive radio wireless networks: A survey Computer Networks 50: 2127-2159.

5. Suman R, Rajeshwar LD, Parmender S (2011) Spectrum Sensing in Cognitive Radio using MIMO Technique. International Journal of Soft Computing and Engineering (IJSCE) 1: 259-265.

6. Mitola J, Maguire GQ Jr (1999) Cognitive radio: making software radios more personal. IEEE Personal Communications 6: 13-18.

7. Ghosh G, Das P, Chatterjee S (2014) Cognitive Radio and Dynamic Spectrum Access - A Study. International Journal of Next-Generation Networks (IJNGN) 6: 43-60.

8. Advanced Research in Information Assurance and Secuity Lab. 
Citation: Singh S, Mushtaq G, Tiwari NK, Singh AP (2015) Cognitive Radio With Software Defined Radio and Mimo for Future Generation Wireless Communication. J Comput Sci Syst Biol 8: 166-169. doi:10.4172/jcsb.1000184

9. Cognitive Radio Tutorial.

10. Algorithms for Cognitive Radio Networks Computer Science Essay.

11. Academia.

12. Software Defined Radio Architectures Systems and Functions.

13. Marja M, Marko H, Miia M, Heli S, Atso H, et al. (2008) Cognitive radio: An intelligent wireless communication system. Research Report Vtt-R-02219-08

14. Chen Z, Wang CX, Hong X, Thompson J (2011) Interference Mitigation for Cognitive Radio Mimo Systems Based on Practical Precoding. IEEE Transaction on Wireless Communications 21: 1-12.

15. Yi H (2010) Nullspace-based secondary joint transceiver scheme for cognitive radio MIMO networks using second-order statistics in IEEE ICC'11 Cape Town South Africa.

16. Gao F, Zhang R, Liang YC, Wang X (2010) Design of learning-based MIMO cognitive radio systems. IEEE Trans Veh Technol 59: 1707-1720.

17. Radio-Electronics. 